Review

\title{
Epigenetics and Medicine
}

Giuseppe Cocco ${ }^{1,}{ }^{*}$, Philipp Amiet ${ }^{2}$

1. Cardiology Office, Marktgasse 10a, CH-4310 Rheinfelden, Switzerland; E-Mail: praxis@cocco.ch

2. Medical Office, Schiffackerweg 2, CH-4310 Rheinfelden, Switzerland; E-Mail: praxiscocco@hin.ch

* Correspondence: Giuseppe Cocco; E-Mail: praxis@cocco.ch

Academic Editor: Joep Geraedts

OBM Genetics

2021, volume 5, issue 3

doi:10.21926/obm.genet.2103133
Received: March 23, 2021

Accepted: July 13, 2021

Published: July 30, 2021

\begin{abstract}
"Epigenetics is the study of how your behaviors and environment can cause changes that affect the way your genes work. Unlike genetic changes, epigenetic changes are reversible and do not change your DNA sequence, but they can change how your body reads a DNA sequence" (https://www.cdc.gov/genomics/disease/epigenetics.htm). Epigenetic interactions, along with the genetic expression in innate cells, change the structure and function of chromatin, and thus, turn the genes on and off. Epigenetic changes influence disease load and resistance and play an important role in health maintenance and almost all medical disorders, and differs significantly with sex and ethnicity. Epigenetic changes may have either positive or detrimental effects on the immune system. They are long-lasting, increase a host's susceptibility to infections and medical pathologies, and affect the efficacy of vaccines. Recent studies have indicated that detrimental epigenetic changes can be mended. Safe and effective mechanisms to reverse detrimental epigenetic scars will have broad medical implications, decrease mortality after infections, and protect the elderly against infections, autoimmune diseases, and cancer. These therapies might be useful for the successful application of vaccines in countries where HIV, parasite infestation, malaria, and other chronic diseases are endemic, and also for a better effect of vaccines in geriatric patients.
\end{abstract}

\section{Keywords}

Epigenetics; immunology; trained immunity; vaccines

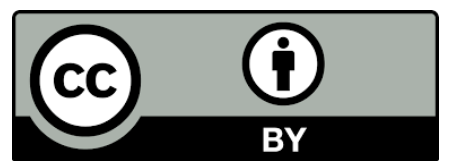

(C) 2021 by the author. This is an open access article distributed under the conditions of the Creative Commons by Attribution License, which permits unrestricted use, distribution, and reproduction in any medium or format, provided the original work is correctly cited. 


\section{Introduction}

There is a constant interaction between the external and internal environments of living organisms and is essential for the normal development and maintenance of health, as well as for influencing disease load and resistance [1, 2]. The immune system, along with its genetic and epigenetic components, is essential for survival. In eukaryotes, DNA forms a stable structure with octamers of histone proteins called nucleosomes and is organized in the cellular nucleus into a three-dimensional structure. This stable structure is known as chromatin. The opening of the chromatin structure affects the accessibility of DNA to transcription factors and RNA polymerase II and is a key factor in determining the rate of mRNA expression [1-4]. The ability to activate a specific promoter during the genetic expression of DNA depends on its location within the chromatin network [1-4]. Antigens induce long-lasting immune responses by the activation of specific innate cells (monocytes and dendritic natural killer cells), which play a central role in immunity. During the lifetime of organisms, they are exposed to dynamic environmental conditions that might trigger chemical changes to activate or silence genes. These changes occur above (in Greek, epi, है $\pi$ ) the DNA and are therefore referred to as epigenetic. This paper reviews the role of epigenetics in common clinical conditions.

\subsection{Definition and Role of Epigenetics in Medicine}

Epigenetics refers to the study of the regulation of gene expression not caused by underlying changes in the DNA sequence. The interactions with the genetic expression of innate cells change the structure and function of chromatin, turn the genes on and off and control various biological phenomena [1-4]. Epigenetic changes influence disease load and resistance and play an important role in health maintenance and almost all medical disorders, such as cardiovascular pathologies [5, $6]$, diabetes mellitus $[7,8]$, rheumatoid arthritis $[1,9,10]$, obesity $[1,2,11]$, and infections $[1-4,12$, 13]. Moreover, some epigenetic changes occur in utero and can affect the risk of developing atherosclerosis later in life [14]. The effects of the immune system differ significantly with sex and ethnicity $[1-4,15]$.

\subsection{Historical Data}

The existence of a specific humoral immunity was detected at the end of the $18^{\text {th }}$ century [16] when it was observed that inoculating rabbits with killed Streptococcus pneumoniae (SP) protected them against infection with SP of the same strain and that infused serum from immune animals protected non-immune rabbits against infection with the same SP strain, but not from infection with other SP strains. Tuberculin is a set of proteins extracted from Mycobacterium tuberculosis (M). The attempt to protect tuberculosis patients with tuberculin was a failure, but it was noted that in these patients, the inoculation of tuberculin caused a delayed, antigen-specific local inflammation characterized by the accumulation of lymphocytes and macrophages. The Bacillus Calmette-Guérin (BCG) is an attenuated strain of Mycobacterium bovis. It was discovered that mice infected with BCG also developed resistance to M. fortuitum and Staphylococcus aureus (SA) [17]. Other experiments proved the existence of non-specific protection against infections. Mice infected with BCG or with 
Brucella melitensis also developed resistance to antigenically unrelated bacteria, such as Listeria [18-20]. Mice infected with BCG were protected against the influenza viruses [21]. Mice infected with Listeria were protected against the extracellular organism SP [22], against Babesia and Plasmodium malariae [23], Leishmania donovani [24], and Toxoplasma [25]. All these experiments demonstrated the existence of non-specific immunity, which was called acquired cellular resistance. It was suggested that this effect was due to an enhanced, non-specific bactericidal capacity of macrophages, which had been activated by antigen-specific stimulation. However, some results of these experiments were not explained. Macrophages from mice that were previously infected with BCG, Brucella, or Listeria maintained enhanced antimicrobial activity after the infecting organisms had been eradicated. Also, the acquired resistance persisted without reintroduction of the sensitizing antigens and was independent of lymphocytes. It has now been established that this immunological protection is due to long-lasting epigenetic changes in macrophages.

\section{Mechanism of Epigenetic Changes}

The genetic expression can be altered by direct and indirect epigenetic pathways [1-4, 26]. The direct pathway can operate in two ways. In type 1 direct pathway, the epigenetic factor directly exerts an effect on the epigenetic enzymes such as DNMTs, HDACs, HATs, HMTs, HDMs, etc., and alters the bioavailability of these enzymes in the cells. In type 2 direct pathway, an epigenetic factor interferes with a biochemical pathway by altering the availability of the metabolites required for constituting an epigenetic tag. Both forms of the direct pathway can result in aberrant or inadequate recruitment of epigenetic tags to non-specific promoters randomly, ultimately establishing an altered epigenetic profile. In the indirect pathway, the epigenetic factor indirectly exerts an effect on the epigenome by first interfering with any signaling pathway in the cell. Acute exposure to the epigenetic factor can cause altered expression of growth factors, receptors, ion channels, etc., resulting in non-homeostatic cellular processes, thus altering the state of the transcriptional machinery (bound or unbound to the promoter/enhancer) and its bioavailability in cells. Chronic exposure to the epigenetic factor might lead to retention of the transcriptional machinery, causing altered gene expression, as well as aberrant recruitment of epigenetic enzymes. This might permanently add or remove epigenetic tags at specific promoters and establish an altered epigenetic profile. Several major epigenetic mechanisms have been described, two of which exert their effect by influencing chromatin compaction [3, 4]. These may be a) by the acetylation or methylation of histones associated with DNA; b) by the methylation of the $5^{\prime}$ cytosine residues of DNA; c) by the release of non-coding RNAs, which also regulate the transcription of DNA; and d) by the transcriptional processes in DNA that might also be modified by methylation of DNA, where the binding of transcription factors to the gene promoters is affected. The described epigenetic changes interfere with the genetic accessibility to promoters and gene transcription in the stimulated innate cells; chromatin accessibility is increased, and innate cells respond faster and stronger to a second stimulus. These long-lasting epigenetic changes are antigen non-specific, cross-protective, and are the basis for trained immunity. Of note, the epigenetic changes may either stimulate or inhibit genetic expression [1-4, 26]. 


\section{Epigenetic Clock}

The strong effects of aging on DNA methylation levels have been known since the late $1960 \mathrm{~s}$, and the first description of an epigenetic clock was presented in 2009 [27, 28]. A set of differentially methylated loci can be used to predict aging and the onset of diseases in aging mouse models. Since age is a fundamental characteristic of most organisms, biological aging clocks and biomarkers of aging find many uses in research. In 2010, a unifying model of aging and the development of complex diseases was proposed, incorporating classical aging theories and epigenetics. Horvath and Raj [28] proposed an epigenetic clock theory of aging with the following tenets: a) biological aging results as an unintended consequence of developmental and maintenance programs, the molecular footprints of which give rise to DNA methylation age estimators, b) the precise mechanisms linking the innate molecular processes (underlying DNAm age) to the decline in tissue function probably are related to intracellular changes (leading to a loss of cellular identity) and subtle changes in cell composition, for example, fully functioning somatic stem cells; and c) at the molecular level, DNAm age is a proximal readout of a collection of innate aging processes that, along with other independent root causes of aging, lead to the deterioration of tissue function. Horvath's clock [27, 28] was defined as an age estimation method based on 353 epigenetic markers of DNA that measure the $\mathrm{CpG}$ dinucleotides of methylated DNA. The Hannum epigenetic clock, published in 2013, consists of 71 markers that accurately estimate age based on blood methylation levels [29]. It allows genetic studies of epigenetic aging, lifestyle factors, obesity and metabolic syndrome, female breast tissue, cancer tissue, trisomy 21, Alzheimer disease-related neuropathology, several neurologic pathologies, HIV-infection, developmental disorders, and menopause, and it is also applied for studying the effects of sex and ethnicity.

\section{Epigenetic Changes}

The existence of epigenetic changes has been proven in several experiments. Within five days of initial exposure to BCG or $\beta$-glucan (BG), innate cells begin to exhibit more robust responses that persist for at least one year, long after the disappearance of the initial stimulus $[6,30]$. Mice vaccinated with BG survived the administration of a lethal dose of SA and SP [30-32], Escherichia coli [33-38], Plasmodium [38], and influenza and yellow fever viruses [2, 36]. Laboratory experiments have proven that trained immunity is independent of lymphocytes, since mice lacking B or T lymphocytes, who received pretreated macrophages and were later infected with BCG and lethal doses of Candida, had robust, antigen-non-specific secondary responses and survived [31, 32]. The epigenetic response is due to increased chromatin accessibility and histone 3 lysine trimethylation (H3K4 me3) in tumor necrosis factor (TNF) and interleukins (IL), such as IL- $\beta$ and IL-6. Consequently, this increases glycolysis, glutaminolysis, and cholesterol synthesis in innate cells, thus affecting the nucleotide-binding oligomerization domain-like receptors, C-type lectin receptors, and other pattern-recognition receptors [4, 13, 31-35]. The strong relationship between epigenetic changes and trained immunity was demonstrated by the finding that the chemical inhibition of methylation blocked BCG-induced epigenetic changes and trained the immune system [32-34]. The experimental results were confirmed in humans. Patients were vaccinated with $B C G$ and three months later were exposed to Candida or SA; their macrophages generated large amounts of IL-6 and TNF [32]. Differentiated innate cells were thought to live only for a few weeks, but trained immunity was still detected one year after the initial epigenetic-mediated sensitization [6, 13]. 
Furthermore, the existence of the long-lasting nature of antigen-non-specific training was proven by detecting terminally differentiated monocytes with an increased antimicrobial capacity of hematopoietic stem cells [34-42]. These experiments demonstrated that macrophages could acquire antigen-non-specific, cross-protective, trained immunity against an array of pathogens. The pathway was associated with increased glycolysis, glutaminolysis, and cholesterol synthesis in innate cells with effects on the nucleotide-binding oligomerization domain-like receptors, C-type lectin receptors, and other pattern-recognition receptors. These changes were due to increased chromatin accessibility and histone 3 lysine trimethylation (H3K4 me3) in TNF. The strong relationship between epigenetic changes and trained immunity was demonstrated by the finding that the chemical inhibition of methylation blocked BCG-induced epigenetic changes and trained the immune system [33-35]. Many epigenetic changes in sepsis have been described in a recent review [3]; lipopolysaccharide (LPS) treated monocytes fail to accumulate active histone marks at the promoters and enhancers of genes associated with the lipid metabolism and phagocytic pathways. Transcriptional inactivity, in response to a second LPS exposure in tolerized macrophages, is accompanied by a failure to deposit active histone marks at promoters of tolerized genes. Of note, exposure to low concentrations of LPS can cause priming, while exposure to high LPS concentrations results in suppressed responses. Briefly, a high bacterial burden in sepsis can lead to a change in the state of chromatin and an associated deviation from steady-state function, a phenomenon known as innate immune memory. The best-characterized outcomes are endotoxin tolerance or trained innate immunity. Trained immunity can have beneficial effects through the priming of macrophages for stronger responses to subsequent infections.

\section{Detrimental Epigenetic Effects}

The epigenetic rewiring of chromatin usually does not induce exuberant or abnormal immunologic effects but might either turn on (stimulate) or turn off (inhibit) genetic expression. Persistent epigenetic changes after an infection or in some pathologies might induce a detrimental antigen non-specific immune suppression and make the host susceptible to some microbial pathogens, thus increasing the risk of future antigen non-specific infections [3-5, 26]. Indeed, detrimental epigenetic changes play a key role in several medical conditions, such as autoimmune pathologies and cancer [1-10, 43-46]. In the early 1900s, it was found that clinical flares of tuberculosis occurred during recovery from measles, and this situation was correlated with suppressed responses to tuberculin on delayed-type hypersensitivity skin tests [4, 42]. Many observations have proven the biological impact of detrimental epigenetic changes. Measles-induced immunosuppression increases the risk of secondary bacterial otitis media, tracheitis, and pneumonia [44-47]. Hepatitis B and C viral infections are associated with an increased risk of SA [4548] and SP infections [49]. Chronic helminthic infestations increase the risk of HIV infection by a factor of 2 to $6[50,51]$ and the risk of tuberculosis by a factor of 4 [52]. Antenatal parasite infestation reduces the efficacy of vaccination in children [53]. Six months after the successful treatment of schistosomiasis in children, reduced immunologic responses to BCG occur in the CD4 ${ }^{+}$ $T$ cells due to detrimental DNA hypermethylation $[54,55]$. The infection-related and non-infectionrelated deaths from all causes are increased after the successful treatment of tuberculosis [54-56]. An infection with SP is followed by a persistent increase in the risk of death that is proportional to the severity of the initial infection [57]. After recovery from sepsis, the risk of death is increased by 
up to $22 \%$ [53], with a prolonged increased risk of secondary bacterial infections [52, 55-61]. It was hypothesized that the initial infection is a marker of increased susceptibility, but it is now known that the suppressed immune response is due to detrimental epigenetic changes in both innate and adaptive immune cells. Persistent detrimental epigenetic changes have been confirmed by finding that two years after effective antiretroviral therapy, HIV-infected patients retain detrimental epigenetic marks in the genes encoding IL-2 and programmed death in CD8 ${ }^{+}$T lymphocytes [54]. Many laboratory experiments have proven the existence of detrimental epigenetic changes. Exposure of monocytes to high LPS concentrations induces a closed chromatin pattern that epigenetically silences the gene encoding IL- 6 with decreased production of IL- 6 and other proinflammatory cytokines on re-stimulation [3, 31, 32]. The monocytes fail to accumulate active histone marks at the promoters and enhancers of genes in the lipid metabolism and phagocytic pathways. Transcriptional inactivity, in response to a second LPS exposure in tolerized macrophages, is accompanied by a failure to deposit active histone marks at the promoters of tolerized genes. Macrophages of mice infected with Escherichia coli or the influenza viruses show epigenetic changes with an antigen non-specific decreased capacity to phagocytize unrelated bacteria for at least six months after the initial infection [57]. In mice, chronic Mycobacterium avium infection induces dysfunction in stem-cell differentiation [60], and on secondary stimulation, their macrophages have a decreased production of TNF [61]. In mice, chronic lymphocytic choriomeningitis virus (LCMV) infection decreases the production of IL-2, interferon- $\gamma$, and TNF in lymphocytes, decreases T-cell proliferative capacity, delays hypersensitivity, reduces antigen non-specific microbial killing capacity, and reduces immunologic activity by inducing DNA hypermethylation at the promoter region of IFNG that inhibits $C D 8^{+}$T-lymphocyte immunity $[62,63] ;$ T lymphocytes show exhausted memory [9, 64-66]. Finally, in animal models of sepsis (12 weeks after ligation of the colon), persistent epigenetic modifications inhibit IL-12 production in dendritic cells, diminish type 1 helper T-lymphocyte immunity, and skew immunity towards a type 2 helper T-lymphocyte response with decreased interferon- $\gamma$ and increased IL-4, IL-5, and IL-13 production [32, 67]. These experimental data provide strong evidence for the argument that long-lasting epigenetic scarring causes detrimental changes in stem cells.

\section{Epigenetics and Aging}

Aging induces detrimental epigenetic changes and reduces new immune memory $[4,8,13,27]$. Immune cells in old people show global DNA hypermethylation and a more closed chromatin pattern at the T-lymphocyte factor 1 . This reduces the memory for the formation of immunity and alters the function of the IL-7 receptor that is required for the survival of memory cells $[4,13,27$, 67]. These changes might explain why the elderly have increased susceptibility to infections, decreased response to immunization, and increased risk of cancer and autoimmune diseases $[1,13$, $27,68]$.

\section{Epigenetics and Medical Pathologies}

The pathogenesis of autoimmune diseases has not been fully elucidated, but through genetic mapping, several candidate variants in autoimmune pathologies have been identified [1-6, 27]. Epigenetic changes interact with DNA transcription factors and play an important role in the pathogenesis of many diseases, such as arteriosclerosis, cancer, type 1 diabetes, multiple sclerosis, 
rheumatoid arthritis, systemic lupus erythematosus, and systemic sclerosis [1-6, 67-74]. Oncologists have established the Cancer Genome Atlas and Gene Expression Omnibus public database repositories to aid in the identification of new therapeutic strategies. Because of the similarities between induced immune suppression in cancer and chronic infections, the tools identified to reverse cancer-induced detrimental epigenetic scars also could reverse detrimental post-infectious epigenetic scars $[28,29,68-75]$.

\section{Mending Detrimental Epigenetic Changes}

A deleterious tolerance after an initial inflammation phase may be reversed. In mice with chronic LCMV infection, the hypomethylating agent decitabine reversed the detrimental DNA hypermethylation marks in $\mathrm{CD}^{+} \mathrm{T}$ lymphocytes and restored immune functionality [18-20,22$25,64]$. In ex vivo studies, exposure to BG human monocytes with detrimental epigenetic chromatin marks decreased responsiveness to lipopolysaccharide and restored the production of TNF [71]. In vivo, the LPS-exposed monocytes were rescued through exposure to BG by inducing early active histone dynamics. Generally, H3K27ac accumulation is accompanied by H3K4 me1 accumulation, mostly at the BG-induced enhancers $[28,30]$. These studies showed that the mechanisms controlling monocyte tolerance could also be reverted in vivo to a more responsive phenotype. However, contrary to this general pattern, LPS-induced active enhancers, associated with inflammatory responses, showed discordance in time with the accumulation of H3K4 me1, which remained at a higher level in LPS-macrophages compared to their levels in naive-macrophages and BGmacrophages [30-32]. In a study [30], more than 3,000 de novo macrophage enhancers were found that were modulated in the opposite direction by BG or LPS exposure. Deposition of H3K27ac and H3K4 me1 at these regions was accelerated by BG exposure and delayed or completely blocked by LPS exposure. Accordingly, expression of genes near these elements was induced by BG, peaking 24 hours post-exposure, while the expression of these genes remained low in the LPS-exposed monocytes. These genes were involved in lipid metabolism and biosynthesis, phagocytosis, and lysosome maturation. The response of macrophages to infections requires a substantial amount of energy with shifts in metabolism and energy production, which changes macrophage polarization to $\mathrm{M} 1$ or $\mathrm{M} 2$ subtypes and establishes trained immunity [30-32].

Immunometabolic processes are abnormal in atherosclerosis [1-6, 13, 69]. Efferocytosis (the removal of inflamed and dying cells by phagocytosis) is recognized as a hallmark of atherosclerosis and is caused in part by the pathologic upregulation of the antiphagocytic signal molecule CD47 [69, $74,75]$. In preclinical studies, CD47 downregulation therapy was shown to reduce atherosclerotic burden and plaque rupture [74], and the humanized anti-CD47 antibody magrolimab might reduce vascular inflammation, as quantified by 18 F-FDG uptake in the carotid arteries $[75,76]$. Some drugs, such as HMG-CoA reductase inhibitors (statins), reduced the risk of adverse cardiovascular effects by counteracting detrimental epigenetic modifications that might have occurred in monocytederived macrophages [77]. In elderly patients, BCG vaccination decreased infections (especially respiratory infections) from all causes and increased cytokine production induced by Candida, and these positive effects were due to the correction of detrimental epigenetic rewiring [78-80]. In contrast, BG partially reversed the LPS-induced tolerance in vitro. Importantly, ex vivo BG treatment of monocytes from volunteers with experimental endotoxemia reinstated their capacity for cytokine production [28]. The tolerance was reversed at the level of distal element histone modification and 
transcriptional reactivation of otherwise unresponsive genes [4, 5]. These findings are important steps toward understanding how the tolerized phenotype might be reversed and ultimately provide the framework for future therapeutic developments in many diseases.

\section{Epigenetics and Vaccines}

Many available vaccines were developed with an exclusive focus on the infecting agents, the vaccines, and the adjuvants, but little attention was given to the vaccinated host [81]. Response to a vaccine depends not only on the nature of the antigen but also on the immune system of the recipient [82]. We have attenuated live and dead vaccines. Examples of live vaccines include yellow fever and polio vaccination. A live vaccination can last a lifetime while a dead vaccination requires regular booster shots throughout life; live vaccines can also be stored without refrigeration [80]. With live vaccines, there is an increased risk for adverse events in individuals with a compromised immune system, such as patients with AIDS, those treated with drugs that prevent the rejection of organ transplantation, or those undergoing chemotherapy [82, 83]. Multiple mRNA platforms for vaccination against infectious diseases and several types of cancer have provided encouraging results and represent a valid alternative to conventional vaccines due to their high potency, capacity for rapid development, and the potential for low-cost production and safe administration [84]. The rapid progress in the development of mRNA vaccines would not have been possible without recent major advances in the areas of innate immune sensing of RNA and in vivo delivery methods. These vaccines have played a central role in the prevention of the COVID-19 infection, and their efficacy was related to the induced epigenetic changes in the recipient [84]. Ideally, vaccinations should simultaneously stimulate classic B-cell and T-lymphocyte adaptive immunity, as well as induce antigen non-specific innate immune training. Vaccination with the specific antigens Rv0125 and Rv119 of M. tuberculosis, together with the adjuvant AS01E, induces robust responses in the innate and adaptive immune cells and provides substantial protection against the progression of tuberculosis [85]. The vaccine MTBVAC might enhance T-lymphocyte immunity and induce epigenetically mediated trained immunity, and a study [86] is assessing its effect in SP. The VPM1002 strain of BCG is bioengineered to lack urease production and includes listeriolysin O, a toxin that enables Listeria to escape the phagosome. Previous administration of a live vaccine augmented humoral responses to vaccines against influenza and hepatitis B viruses, SP, and meningococcus [13]. However, in children, especially in girls, the administration of some dead vaccines was shown to increase the risk of unrelated, antigen non-specific infections and reduced the efficacy of some live vaccines. On the other hand, previous administration of a live vaccine augmented the humoral responses to vaccines against influenza and hepatitis B viruses, meningococcus, and SP [86]. Also, a study with adolescents [13] found that in the year after BCG vaccination, the risk of upper respiratory tract infections was reduced by $72 \%$. In the elderly, BCG vaccination was shown to induce positive epigenetic changes, consequently augmenting the antigen non-specific innate immunity with a $75 \%$ decrease in respiratory infections. Follow-ups from eight months to five years have proven that BCG, oral polio, and measles vaccines decreased non-targetrelated infections and mortality from all causes $[78,79]$. Of note, the effect of BCG vaccination inhibited systemic inflammation in a sex-dependent manner [88]. Ongoing studies are assessing whether BCG, oral polio, and measles vaccines might exert an antigen-unrelated immunity and decrease non-specific infections during the COVID-19 pandemic [84]. 


\section{Discussion}

Modern studies have outlined the key role of epigenetic changes for immunity in innate and adaptive immune cells in all living organisms. In humans epigenetic effects differ significantly with the sex, ethnicity, and age. Antigen-specific and non-antigen-specific mechanisms interact and play a central role in health and diseases. Epigenetic changes may be both beneficial and detrimental. Identifying the metabolic processes that drive epigenetic changes would help to determine the methods to interact with the system. Research is walking on the long learning road of epigenetics. At least some detrimental epigenetic changes can be mended. Innate cells may be reprogrammed to recover from detrimental changes due to aging and infections. The effect of vaccines may depend on previous pathologies, the age of the person, the type of the vaccine, and the order in which they are administered. Safe and effective mechanisms to reverse detrimental epigenetic scars will have broad medical implications, decrease mortality after infections, and protect the elderly against infections, autoimmune diseases, and cancer. These therapies might be useful for the successful application of vaccines in countries where chronic infections are endemic and also for a better effect of vaccines in geriatric patients.

\section{Acknowledgments}

The authors thank Mrs. Jacqueline Bugmann for her secretarial work and typing the manuscript.

\section{Author Contributions}

G. Cocco choose the references from the literatue, wrote the manuscript and corresponded with reviwers and editors; Ph. Amiet discussed the manuscript and the recommended changes. He also read the text several times for mistakes, omissions etc.

\section{Competing Interests}

The authors have declared that no competing interests exist.

\section{References}

1. Kanherkar RR, Bhatia-Dey N, Csoka AB. Epigenetics across the human lifespan. Front Cell Dev Biol. 2014; 2: 49.

2. Zhao YZ, Forst CV, Sayegh CE, Wang I-M, Yang X, Zhang B. Molecular and genetic inflammation networks in major human diseases. Mol Biosyst. 2016; 12: 2318-2341.

3. Cross D, Drury R, Hill J, Pollard AJ. Epigenetics in Sepsis: Understanding its role in endothelial dysfunction, immunusupression, and potential therapeutics. Front Immunol. 2019; 10: 1363.

4. DiNardo AR, Netea MG, Musher DM. Postinfectious epigenetic immune modifications - a double- edged sword. N Engl J Med. 2021; 384: 261-270.

5. Willerson JT, Ridker PM. Inflammation as a cardiovascular risk factor. Circulation. 2004; 109: 210.

6. Cocco G, Jerie P, Amiet P, Pandolfi S. Inflammation in heart failure: Known knowns and unknown unknowns. Expert Opin Pharmacother. 2017; 18: 1225-1233. 
7. Duncan BB, Schmidt MI, Pankowet S, Ballantyne CM, Couper D, Vigo A, et al. Low-grade systemic inflammation and the development of type 2 diabetes: The atherosclerosis risk in communities study. Diabetes. 2003; 52: 1799-1805.

8. Napoli C, Benincasa G, Schiano C, Salvatore M. Differential epigenetic factors in the prediction of cardiovascular risk in diabetic patients. Eur Heart J Cardiovasc Pharmacother. 2020; 6: 239247.

9. Wu HJ, Zhao M, Yoshimura A, Chang C, Lu QJ. Critical link between epigenetics and transcription factors in the induction of autoimmunity: A comprehensive review. Clin Rev Allergy Immunol. 2016; 50: 333-344.

10. Gonzalez-Jaramillo V, Portilla-Fernandez E, Glisic M, Voortman T, Ghanbari M, Bramer W, et al. Epigenetics and inflammatory markers: A systematic review of the current evidence. Int J Inflam. 2019; 2019: 1-14.

11. Alegría-Torres JA, Baccarelli A, Bollati V. Epigenetics and lifestyle. Epigenomics. 2011; 3: 267277.

12. Crimi E, Benincasa G, Cirri S, Mutesi R, Faenza M, Napoli C. Clinical epigenetics and multidrugresistant bacterial infections: Host remodelling in critical illness. Epigenetics. 2020; 10: 10211034.

13. Crimi E, Benincasa G, Figueroa-Marrero N, Galdiero M, Napoli C. Epigenetic susceptibility to severe respiratory viral infections and its therapeutic implications: $\mathrm{A}$ narrative review. $\mathrm{Br} \mathrm{J}$ Anaesth. 2020; 125: 1002-1017.

14. de Nigris F, Cacciatore F, Mancini FP, Vitale DF, Mansueto G, D'Armiento FP, et al. Epigenetic hallmarks of fetal early atherosclerosis in humans. JAMA Cardiol. 2018: 3: 1184-1191.

15. Hartman RJ, Sarah E, Huisman SE, Hester M, den Ruijter HM. Sex differences in cardiovascular epigenetics-a systematic review. Biol Sex Differ. 2018; 9: 1-8.

16. Gray G, Musher DM. The history of pneumococcal disease. Washington, DC: ASM Press; 2008.

17. Elberg SS, Schneider P, Fong J. Cross-immunity between Brucella melitensis and Mycobacterium tuberculosis. Intracellular behavior of Brucella melitensis in monocytes from vaccinated animals. J Exp Med. 1957; 106: 545-554.

18. Dubos RJ, Schaedler RW. Effects of cellular constituents of mycobacteria on the resistance of mice to heterologous infections I. Protective effects. J Exp Med. 1957; 106: 703-717.

19. Mackaness GB. Cellular resistance to infection. J Exp Med. 1962; 116: 381-406.

20. Mackaness GB. The immunological basis of acquired cellular resistance. J Exp Med. 1964; 120: 105-120.

21. Spencer JC, Ganguly R, Waldman RH. Nonspecific protection of mice against influenza virus infection by local or systemic immunization with Bacille Calmette-Guérin. J Infect Dis. 1977; 136: 171-175.

22. Musher DM, Ratzan KR, Weinstein L. The effect of Listeria monocytogenes on resistance to pneumococcal infection. Proc Soc Exp Biol Med. 1970; 135: 557-560.

23. Clark IA, Allison AC, Cox FE. Protection of mice against Babesia and Plasmodium with BCG. Nature. 1976; 259: 309-311.

24. Murray HW, Rubin BY, Rothermel CD. Killing of intracellular Leishmania donovani by lymphokine-stimulated human mononuclear phagocytes: Evidence that interferon-gamma is the activating lymphokine. J Clin Invest. 1983; 72: 1506-1510. 
25. Remington JS, Krahenbuhl JL, Mendenhall JW. A role for activated macrophages in resistance to infection with Toxoplasma. Infect Immun. 1972; 6: 829-834.

26. Fanucchi S, Fok ET, Dalla E, Shibayama Y, Börner K, Chang EY, et al. Immune genes are primed for robust transcription by proximal long noncoding RNAs located in nuclear compartments. Nat Genet. 2019; 51: 138-150.

27. Epigenetic clock [Internet]. Available from: https://en.wikipedia.org/wiki/Epigenetic clock.

28. Horvath S, Raj K. DNA methylation-based biomarkers and the epigenetic clock theory of ageing. Nat Rev Genet. 2018; 19: 371-384.

29. Hannum G, Guinney J, Zhao L, Zhang L, Hughes G, Sadda S, et al. Genome-wide methylation profiles reveal quantitative views of human aging rates. Mol Cell. 2013; 49: 359-367.

30. Novakovic B, Habibi E, Wang S-Y, Arts RJ, Davar R, Megchelenbrink W, et al. $\beta$-Glucan reverse the epigenetic state of LPS-induced immunological tolerance. Cell. 2016; 167: 1354-1368.e14.

31. Arts RJ, Carvalho A, La Rocca C, Palma C, Rodrigues F, Silvestre R, et al. Immunometabolic pathways in BCG-induced trained immunity. Cell Rep. 2016;17: 2562-2571.

32. Saeed S, Quintin J, Kerstens HH, Rao NA, Aghajanirefah A, Matarese F, et al. Epigenetic programming of monocyte-to-macrophage differentiation and trained innate immunity. Science. 2014; 345: 1251086.

33. Verma D, Parasa VR, Raffetseder J, Martis M, Mehta RB, Netea M, et al. Anti-mycobacterial activity correlates with altered DNA methylation pattern in immune cells from BCG-vaccinated subjects. Sci Rep. 2017; 7: 1-10.

34. Kleinnijenhuis J, Quintin J, Preijers F, Joosten LA, Ifrim DC, Saeed S, et al. Bacille Calmette-Guerin induces NOD2-dependent nonspecific protection from reinfection via epigenetic reprogramming of monocytes. Proc Natl Acad Sci USA. 2012; 109: 17537-17542.

35. Cheng SC, Quintin J, Cramer RA, Shepardson KM, Saeed S, Kumar V, et al. mTOR- and HIF-1 $\alpha-$ mediated aerobic glycolysis as metabolic basis for trained immunity. Science. 2014; 345: 1250684.

36. Arts RJ, Moorlag SJ, Novakovic B, Li Y, Wang SY, Oosting M, et al. BCG vaccination protects against experimental viral infection in humans through the induction of cytokines associated with trained immunity. Cell Host Microbe. 2018; 23: 89-100.e5.

37. Kaufmann E, Sanz J, Dunn JL, Laura E, Mendonça LE, Pacis A, et al. BCG educates hematopoietic stem cells to generate protective innate immunity against tuberculosis. Cell. 2018; 172: 176190.e19.

38. Walk J, de Bree LC, Graumans W, Stoter R, van Gemert G-J, van de Vegte-Bolmer M, et al. Outcomes of controlled human malaria infection after BCG vaccination. Nat Commun. 2019; 10 : 1-8.

39. Kleinnijenhuis J, Quintin J, Preijers F, Benn CS, Joosten LA, Jacobs C, et al. Long-lasting effects of BCG vaccination on both heterologous Th1/Th17 responses and innate trained immunity. J Innate Immun. 2014; 6: 152-158.

40. Mitroulis I, Ruppova K, Wang B, Chen L-S, Grzybek M, Grinenko T, et al. Modulation of myelopoiesis progenitors is an integral component of trained immunity. Cell. 2018; 172: 147161.e12.

41. de Laval B, Maurizio J, Kandalla PK, Brisou G, Simonnet L, Huber C, et al. C/EBPß-dependent epigenetic memory induces trained immunity in hematopoietic stem cells. Cell Stem Cell. 2020; 26: 657-674.e8. 
42. Cirovic B, de Bree LC, Groh L, Blok BA, Chann J, van der Velden WJ, et al. BCG vaccination in humans elicits trained immunity via the hematopoietic progenitor compartment. Cell Host Microbe. 2020; 28: 322-334.e5.

43. Zhang $Q$, Zhao K, Shen QC, Han YM, Gu Y, Li X, et al. Tet2 is required to resolve inflammation by recruiting Hdac2 to specifically repress IL-6. Nature. 2015; 525: 389-393.

44. Hu LL, Yu YF, Huang HJ, Fan HT, Hu L, Yin CY, et al. Epigenetic regulation of interleukin 6 by histone acetylation in macrophages and its role in paraquat-induced pulmonary fibrosis. Front Immunol. 2017; 7: 696.

45. Bannister S, Messina NL, Novakovic B, Curtis N. The emerging role of epigenetics in the immune response to vaccination and infection: A systematic review. Epigenetics. 2020; 15: 555-593.

46. Turk JL. Von Pirquet, allergy and infectious diseases: A review. J R Soc Med. 1987; 80: 31-33.

47. Perry RT, Halsey NA. The clinical significance of measles: A review. J Infect Dis. 2004; 189: S4S16.

48. Kaka AS, Filice GA, Kuskowski M, Musher DM. Does active hepatitis $C$ virus infection increase the risk for infection due to Staphylococcus aureus? Eur J Clin Microbiol Infect Dis. 2017; 36: 1217-1223.

49. Marrie TJ, Tyrrell GJ, Majumdar SR, Eurich DT. Concurrent infection with hepatitis $C$ virus and Streptococcus pneumoniae. Emerg Infect Dis. 2017; 23: 1118-1123.

50. Downs JA, van Dam GJ, Changalucha JM, Corstjens PL, Peck RN, de Dood CJ, et al. Association of schistosomiasis and HIV infection in Tanzania. Am J Trop Med Hyg. 2012; 87: 868-873.

51. Kroidl I, Saathoff E, Maganga L, Makunde WH, Hoerauf A, Geldmacher C, et al. Effect of Wuchereria bancrofti infection on HIV incidence in southwest Tanzania: A prospective cohort study. Lancet. 2016; 388: 1912-1920.

52. Elias D, Mengistu G, Akuffo $H$, Britton S. Are intestinal helminths risk factors for developing active tuberculosis? Trop Med Int Health. 2006; 11: 551-558.

53. Labeaud $A D$, Malhotra I, King MJ, King CL, King $\mathrm{CH}$. Do antenatal parasite infections devalue childhood vaccination? PLoS Negl Trop Dis. 2009; 3: e442.

54. DiNardo AR, Nishiguchi T, Mace EM, Rajapkshe K, Mtewa G, Kay A, et al. Schistosomiasis induces persistent DNA methylation and tuberculosis-specific immune changes. J Immunol. 2018; 201: 124-133.

55. van der Poll T, van de Veerdonk FL, Scicluna BP, Netea MG. The immunopathology of sepsis and potential therapeutic targets. Nat Rev Immunol. 2017; 17: 407-420.

56. Roquilly A, Jacqueline C, Davieau M, Mollé A, Sadek A, Fourgeux C, et al. Alveolar macrophages are epigenetically altered after inflammation, leading to long-term lung immunoparalysis. Nat Immunol. 2020; 21: 636-648.

57. Sandvall B, Rueda AM, Musher DM. Long-term survival following pneumococcal pneumonia. Clin Infect Dis. 2013; 56: 1145-1146.

58. Prescott HC, Osterholzer JJ, Langa KM, Angus DC, Iwashyna TJ. Late mortality after sepsis: Propensity matched cohort study. BMJ. 2016; 353: i2375.

59. Matatall KA, Jeong M, Chen SY, Sun DQ, Chen FJ, Mo QX, et al. Chronic infection depletes hematopoietic stem cells through stress-induced terminal differentiation. Cell Rep. 2016; 17: 2584-2595. 
60. Yáñez A, Hassanzadeh-Kiabi N, Ng MY, Megíaset J, Subramanian A, Liu GY, et al. Detection of a TLR2 agonist by hematopoietic stem and progenitor cells impacts the function of the macrophages they produce. Eur J Immunol. 2013; 43: 2114-2125.

61. Ahmed R, Salmi A, Butler LD, Chiller JM, Oldstone MB. Selection of genetic variants of lymphocytic choriomeningitis virus in spleens of persistently infected mice: Role in suppression of cytotoxic T lymphocyte response and viral persistence. J Exp Med. 1984; 160: 521-540.

62. Bengsch B, Johnson AL, Kurachi M, Odorizzi PM, Pauken KE, Attanasio J, et al. Bioenergetic insufficiencies due to metabolic alterations regulated by the inhibitory receptor PD-1 are an early driver of $\mathrm{CD}^{+} \mathrm{T}$ cell exhaustion. Immunity. 2016; 45: 358-373.

63. Miller BC, Sen DR, Al Abosy R, Bi K, Virkud YV, LaFleur MW, et al. Subsets of exhausted CD8 ${ }^{+} \mathrm{T}$ cells differentially mediate tumor control and respond to checkpoint blockade. Nat Immunol. 2019; 20: 326-336.

64. Yao C, Sun H-W, Lacey NE, Ji Y, Moseman EA, Shih HY, et al. Single-cell RNA-seq reveals TOX as a key regulator of $C D 8^{+} T$ cell persistence in chronic infection. Nat Immunol. 2019; 20: 890-901.

65. Wen HT, Dou YL, Hogaboam CM, Kunkel SL. Epigenetic regulation of dendritic cell-derived interleukin-12 facilitates immunosuppression after a severe innate immune response. Blood. 2008; 111: 1797-1804.

66. Cocco G. Immune senescence and Covid-19 pandemic. OBM Geriatrics. 2020; 4: 4.

67. Seo H, Chen J, González-Avalos E, Samaniego-Castruita D, Das A, Wang YH, et al. TOX and TOX2 transcription factors cooperate with NR4A transcription factors to impose $\mathrm{CD}^{+} \mathrm{T}$ cell exhaustion. Proc Natl Acad Sci USA. 2019; 116: 12410-12415.

68. Bates SE. Epigenetic therapies for cancer. N Engl J Med. 2020; 383: 650-663.

69. Stein S. Co-repressing immunometabolic processes in atherosclerosis. Cardiovasc Med. 2021: 24: w10043.

70. Benn CS, Fisker AB, Rieckmann A, Sørup S, Aaby P. Vaccinology: Time to change the paradigm? Lancet Infect Dis. 2020; 20: e274-e283.

71. Kojima Y, Volkmer J-P, McKenna K, Civelek M, Lusis AJ, Miller CL, et al. CD47-blocking antibodies restore phagocytosis and prevent atherosclerosis. Nature. 2016; 536: 86-90.

72. Kojima Y, Weissman IL, Leeper NJ. The role of efferocytosis in atherosclerosis. Circulation. 2017; 135: 476-489.

73. Advani R, Flinn I, Popplewell L, Forero A, Bartlett NL, Ghosh N, et al. CD47 blockade by Hu5F9G4 and rituximab in non-Hodgkin's lymphoma. N Engl J Med. 2018; 379: 1711-1721.

74. Libby P, Ridker PM, Maseri A. Inflammation and atherosclerosis. Circulation. 2002; 105: 11351143.

75. Ghoneim HE, Fan YP, Moustaki A, Abdelsamed HA, Dash P, Dogra P, et al. De novo epigenetic programs inhibit PD-1 blockade-mediated T cell rejuvenation. Cell. 2017; 170: 142-157.e19.

76. Jarr K-U, Nakamoto R, Doan BH, Kojima Y, Weissman IL, Advani RH, et al. Effect of CD47 blockade on vascular inflammation. N Engl J Med. 2021; 384: 382-383.

77. Tawakol A, Fayad ZA, Mogg R, Alon A, Klimas MT, Dansky H, et al. Intensification of statin therapy results in a rapid reduction in atherosclerotic inflammation: Results of a multicenter fluorodeoxyglucose-positron emission tomography/computed tomography feasibility study. J Am Coll Cardiol. 2013; 62: 909-917. 
78. Tarancón R, Domínguez-Andrés J, Uranga S, Ferreira AV, Groh LA, Domenech $M$, et al. New live attenuated tuberculosis vaccine MTBVAC induces trained immunity and confers protection against experimental lethal pneumonia. PLoS Pathgl. 2020; 16: e1008404.

79. Giamarellos-Bourboulis EJ, Tsilika M, Moorlag S, Antigone NA, Kotsaki A, Domínguez-Andrés J, et al. Activate: Randomized clinical trial of BCG vaccination against infection in the elderly. Cell. 2020; 183: 315-323.e9.

80. Kaufmann SH. Vaccination against tuberculosis: Revamping BCG by molecular genetics guided by immunology. Front Immunol. 2020; 11: 316.

81. Chakrabarti P. Living versus dead: The pasteurian paradigm and imperial vaccine research. Bull Hist Med. 2010; 84: 387-423.

82. de St Groth SF, Webster RG. Disquisitions on original antigenic sin. II. Proof in lower creatures. J Exp Med. 1966; 124: 347-361.

83. Mantovani A, Netea MG. Trained innate immunity, epigenetics, and Covid-19. N Engl J Med. 2020; 383: 1078-1080.

84. Pardi N, Hogan MJ, Porter FW, Weissman D. mRNA vaccines - a new era in vaccinology. Nat Rev Drug Discov. 2018; 17: 261-279.

85. Nemes E, Geldenhuys H, Rozot V, Rutkowski KT, Rantangee F, Bilek BN, et al. Prevention of M. tuberculosis infection with H4:IC31 vaccine or BCG revaccination. N Engl J Med. 2018; 379: 138149.

86. Tait DR, Hatherill M, van der Meeren $O$, Ginsberg AM, van Brakel E, Salaun B, et al. Final analysis of a trial of M72/AS01 $\mathrm{E}$ vaccine to prevent tuberculosis. New Engl J Med. 2019; 381: 2429-2439.

87. Márquez EJ, Chung C-H, Marches R, Rossi RJ, Nehar-Belaid D, Eroglu A, et al. Sexual-dimorphism in human immune system aging. Nat Commun. 2020; 11: 1-17.

88. Koeken VA, de Bree LC, Mourits VP, Moorlag SJ, Walk J, Cirovic B, et al. BCG vaccination in humans inhibits systemic inflammation in a sex-dependent manner. J Clin Invest. 2020; 130: 5591-5602.

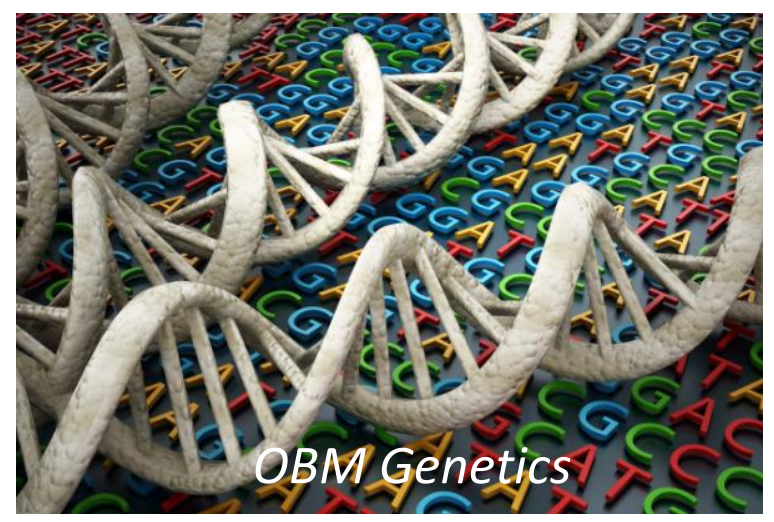

Enjoy OBM Genetics by:

1. Submitting a manuscript

2. Joining in volunteer reviewer bank

3. Joining Editorial Board

4. Guest editing a special issue

For more details, please visit: http://www.lidsen.com/journals/genetics 